\title{
TRIC-B channels display labile gating: evidence from the TRIC-A knockout mouse model
}

\author{
Elisa Venturi • Antoni Matyjaszkiewicz • \\ Samantha J. Pitt • Krasimira Tsaneva-Atanasova • \\ Miyuki Nishi • Daiju Yamazaki • Hiroshi Takeshima • \\ Rebecca Sitsapesan
}

Received: 8 November 2012 /Revised: 24 January 2013 /Accepted: 11 February 2013 / Published online: 7 March 2013

(C) The Author(s) 2013. This article is published with open access at Springerlink.com

\begin{abstract}
Sarcoplasmic/endoplasmic reticulum (SR) and nuclear membranes contain two related cation channels named TRIC-A and TRIC-B. In many tissues, both subtypes are co-expressed, making it impossible to distinguish the distinct single-channel properties of each subtype. We therefore incorporated skeletal muscle SR vesicles derived from Tric- $a$-knockout mice into bilayers in order to characterise the biophysical properties of native TRIC-B without possible misclassification of the channels as TRIC-A, and without potential distortion of functional properties by detergent purification protocols. The native TRIC-B channels were
\end{abstract}

Electronic supplementary material The online version of this article (doi:10.1007/s00424-013-1251-y) contains supplementary material, which is available to authorized users.

E. Venturi $\cdot$ A. Matyjaszkiewicz $\cdot$ R. Sitsapesan $(\bowtie)$

School of Physiology \& Pharmacology,

NSQI and Bristol Heart Institute, University of Bristol,

Bristol BS8 1TD, UK

e-mail: r.sitsapesan@bristol.ac.uk

A. Matyjaszkiewicz $\cdot \mathrm{K}$. Tsaneva-Atanasova

Department of Engineering Mathematics, University of Bristol, Bristol BS8 1TR, UK

\author{
A. Matyjaszkiewicz \\ Bristol Centre for Complexity Sciences, University of Bristol, \\ Bristol BS8 1TR, UK
}

\section{S. J. Pitt}

School of Medicine, University of St Andrews,

St Andrews KY16 9TF, UK

\section{Nishi $\cdot$ D. Yamazaki $\cdot H$. Takeshima $(\square)$}

Graduate School of Pharmaceutical Sciences and Center

for the Promotion of Interdisciplinary Education and Research,

Kyoto University, Kyoto 606-8501, Japan

e-mail: takeshim@pharm.kyoto-u.ac.jp ideally selective for cations. In symmetrical $210 \mathrm{mMK}^{+}$, the maximum (full) open channel level $(199 \mathrm{pS})$ was equivalent to that observed when wild-type SR vesicles were incorporated into bilayers. Analysis of TRIC-B gating revealed complex and variable behaviour. Four main subconductance levels were observed at approximately $80 \%$ (161 pS), $60 \%$ (123 pS), $46 \%$ (93 pS), and $30 \%(60 \mathrm{pS})$ of the full open state. Seventy-five percent of the channels were voltage sensitive with Po being markedly reduced at negative holding potentials. The frequent, rapid transitions between TRIC-B sub-conductance states prevented development of reliable gating models using conventional singlechannel analysis. Instead, we used mean-variance plots to highlight key features of TRIC-B gating in a more accurate and visually useful manner. Our study provides the first biophysical characterisation of native TRIC-B channels and indicates that this channel would be suited to provide counter current in response to $\mathrm{Ca}^{2+}$ release from the SR. Further experiments are required to distinguish the distinct functional properties of TRIC-A and TRIC-B and understand their individual but complementary physiological roles.

Keywords Sarcoplasmic reticulum · Ion channels . Excitation-contraction coupling $\cdot \mathrm{Ca}^{2+}$ release

\section{Introduction}

The TRIC channel is an intracellular ion channel that forms a homo-trimeric assembly with a bullet-shaped structure $[20,32]$. Two TRIC subtypes, namely TRIC-A and TRIC$\mathrm{B}$, are found on endo/sarcoplasmic reticulum (ER/SR) and nuclear membranes in animal cells. TRIC-A is especially 
abundant in excitable tissues, such as cardiac and skeletal muscle, while TRIC-B is found in most tissues [32]. We have previously demonstrated that recombinant, purified TRIC-A and TRIC-B proteins behave as monovalent cation channels when reconstituted into artificial membranes and that they display many similar biophysical properties to the native $\mathrm{SR} \mathrm{K}^{+}$channels that are observed when SR vesicles from skeletal and cardiac muscle are incorporated into bilayers [7, 15, 16, 26, 27].

The physiological role of TRIC channels is not yet fully established. There is good evidence, however, to support the hypothesis that they act as a counter current pathway, enabling sufficient monovalent cation flux to buffer the loss of positive charge that results during SR $\mathrm{Ca}^{2+}$-release events $[30,32]$. Much of this evidence comes from studying the pathology of the Tric-knockout mouse models [30-33]. In the Tric- $b$-knockout mouse, the alveolar epithelial cells exhibit inadequate $\mathrm{Ca}^{2+}$-release from overloaded ER stores, and this causes the mice to die in respiratory failure following birth [30]. In mutant mice lacking both TRIC-A and TRIC-B, the cardiac SR becomes swollen and cardiac arrest is observed after embryonic day E9.5 [32]. Although the Tric- $a$-knockout (Tric- $a$-KO) mouse is viable, the ability of the skeletal muscle cells to respond to stress is defective, and this is revealed by instability in the $\mathrm{Ca}^{2+}$-release process [33]. Moreover, this knockout mouse develops hypertension due to impaired hyperpolarization signalling evoked by $\mathrm{Ca}^{2+}$ spark/release in vascular smooth muscle [31].

The survival of the Tric- $a$-KO mice provides us with the opportunity of isolating SR membranes from tissues which express only the TRIC-B subtype. This is important because although we have previously investigated the single-channel properties of recombinant TRIC-A and TRIC-B channels purified from cDNA-transfected yeast cells [20], it is well known that purification procedures can alter the functional properties of ion channels [11, 13, 14, 29]. At the same time, it is impossible to properly explore the individual singlechannel characteristics of native TRIC-A and TRIC-B channels since they are expressed together in most tissues. Our work with purified TRIC channel subtypes shows that they share certain biophysical properties such as selectivity for monovalent cations, activation by positive holding potentials and complicated sub-conductance state gating behaviour. These similarities make it difficult to definitively distinguish between TRIC-A and TRIC-B channels after incorporating native SR into artificial membranes. In this study, we have therefore isolated SR vesicles from the skeletal muscle of TRIC-A deficient mice. This has allowed us to characterise the basic single-channel properties of TRIC-B channels without interference from the possible co-incorporation of TRIC-A channels into the bilayers. This study provides the first description of the single-channel behaviour of native mouse TRIC-B channels. In future studies, it will be important to investigate the functional differences between TRIC-B and TRIC-A channels as such experiments will provide essential data for understanding their complementary yet distinct physiological roles.

\section{Materials and methods}

Preparation of sucrose gradient fractions

As previously described, a discontinuous sucrose gradient was used to separate sarcolemmal membrane vesicles from the various SR membrane fractions, including light SR (LSR) and heavy SR (HSR) from wild-type (WT) mouse skeletal and cardiac muscle and from Tric- $a$-KO mouse skeletal tissue $[23,24]$. For each tissue, the various membrane fractions were aliquoted and stored at $-80^{\circ} \mathrm{C}$.

Planar phospholipid bilayer techniques

Recombinant TRIC-B channels or LSR or HSR vesicles from WT and Tric- $a-\mathrm{KO}$ tissues were reconstituted into planar phosphatidylethanolamine lipid bilayers as described previously [24]. Recording solutions were symmetrical, unless otherwise indicated, and contained $210 \mathrm{mM}$ KPIPES, $10 \mu \mathrm{M}$ free $\mathrm{Ca}^{2+}, \mathrm{pH} 7.2$. Briefly, after forming a gradient of $\mathrm{K}^{+}$between the two compartments, recombinant proteins or SR vesicles were added to the cis (cytosolic) side of the bilayer. After channel incorporation, the cis chamber was perfused with fresh KPIPES solution to restore symmetrical conditions. The trans (luminal) chamber was held at ground, and the cis chamber was clamped at potentials relative to ground. Experiments were performed at room temperature $\left(22 \pm 2{ }^{\circ} \mathrm{C}\right)$. The free $\left[\mathrm{Ca}^{2+}\right]$ and $\mathrm{pH}$ of the solutions were maintained constant during the experiment and were determined using a $\mathrm{Ca}^{2+}$ electrode (Orion 93-20) and a Ross-type $\mathrm{pH}$ electrode (Orion 81-55) as previously described [24]. After addition of $10 \mathrm{mM}$ EGTA to the cis chamber without further addition of $\mathrm{Ca}^{2+}$, the approximate free $\left[\mathrm{Ca}^{2+}\right]$ was calculated using the program MaxChelator (www.stanford.edu/ $\sim$ cpatton/ maxc.html) to be $<1 \mathrm{nM}$.

\section{Criteria for defining TRIC-B channels}

Addition to the cis chamber, of LSR or HSR vesicles from Tric- $a-\mathrm{KO}$ tissue, in the presence of a $\mathrm{KCl}$ gradient, led to the rapid incorporation of both cationic and anionic channels. In symmetrical solutions of KPIPES, only two distinct types of $\mathrm{K}^{+}$current fluctuations were routinely observed: (1) those we describe as TRIC-B currents (observed in $100 \%$ of bilayer experiments), characterised by full open channel conductance of $200 \mathrm{pS}$ but always observed to visit multiple sub-conductance states as described below; (2) those 
corresponding to currents through RyR channels (singlechannel conductance of approximately 700 pS). RyR channels incorporated less frequently into the bilayer when LSR rather than HSR fractions were used, and for this reason, only TRIC-B channels from the LSR fraction was used. Supplementary Fig. 1 illustrates a typical recording where both an RyR and a TRIC-B channel from the LSR have incorporated into the bilayer demonstrating that the singlechannel characteristics of these two channels are very different and could not be confused. We only used those recordings where no RyR channels were observed and where no leak in the baseline was detected. It is possible that there may be contamination of our SR membrane fractions by sarcolemmal membrane vesicles. However, when skeletal muscle sarcolemmal vesicles are fused into bilayers, fusion events are slower and less reproducible in terms of the type of current fluctuations that are observed. Fusion of sarcolemmal membranes with a bilayer occasionally led to the appearance of a $\mathrm{Ca}^{2+}$-activated $\mathrm{K}^{+}$channel of conductance of approximately $250 \mathrm{pS}$ in symmetrical $210 \mathrm{~K}^{+}$solutions, but we did not observe this channel after fusion of LSR membrane vesicles with the bilayer. If this channel had incorporated during fusion of LSR vesicles, we would have been able to distinguish it from a TRIC channel on the basis of conductance, sub-conductance gating behaviour and $\mathrm{Ca}^{2+}$ dependence. The $\mathrm{Ca}^{2+}$ dependence of the $\mathrm{Ca}^{2+}$-activated $\mathrm{K}^{+}$ channel is well known (for example $[12,17]$ ). In our hands, the $\mathrm{Ca}^{2+}$-activated $\mathrm{K}^{+}$channel that we observe from the sarcolemmal fraction is closed by reducing the cis free $\left[\mathrm{Ca}^{2+}\right]$ to $0.1 \mu \mathrm{M}$ (results not shown), whereas TRIC-B is not regulated by $\mathrm{Ca}^{2+}$ in this fashion (see below).

Data acquisition and analysis of single-channel recordings

Single-channel recordings were digitised at $100 \mathrm{kHz}$ and recorded on a computer hard drive using WinEDR 3.05 software (John Dempster, Strathclyde University, UK). Current-voltage relationships were constructed using current amplitudes, which were measured using manually controlled cursors in WinEDR 3.05. The conductance values were obtained by linear regression (Graphpad Prism 4, La Jolla, USA). We consistently observed four sub-conductance states at +30 and $-30 \mathrm{mV}$. At higher holding potentials or improved resolution of the single-channel events, it is likely that more sub-conductance states would be detected. Using our initial sub-conductance state amplitude measurements, single-channel current fluctuations were idealised using the segmental k-means (SKM) algorithm [21] in the QuB software suite (State University of New York, Buffalo, USA). Before idealisation, traces were digitally filtered (Gaussian Filter with cut-off frequency of $1 \mathrm{kHz}$ ) and re-sampled at $10 \mathrm{kHz}$. A dead time of $600 \mu \mathrm{s}$ was imposed on each idealisation. A six-state Markov model was constructed with identical fully connected topology, where states consisted of the closed, open and four subconductance states using our initial estimates of the current amplitudes of the conductance states. After building the model, the Viterbi algorithm [2, 5] was applied in order to estimate the best fit to our data based on the log-likelihood function value. The parameters of the model were then used to plot amplitude probability density functions which were superimposed on the all-points amplitude histogram. The idealisations obtained with the SKM method were also superimposed on the single-channel traces to visually examine the accuracy of the method. The probability of dwelling in each of the states (closed, open and four sub-conductance states) was determined from the SKM idealisation of continuous recordings of 2-3 min at holding potentials of +30 and $-30 \mathrm{mV}$. To check whether a single channel only was present in the bilayer, high positive holding potentials were applied for short periods of time to maximally activate the channel at the end of the experiment.

Average current, I, was calculated using the following equation:

$I=P_{\text {full }} i_{\text {full }}+P_{\mathrm{S} 1} i_{\mathrm{S} 1}+P_{\mathrm{S} 2} i_{\mathrm{S} 2}+P_{\mathrm{S} 3} i_{\mathrm{S} 3}+P_{\mathrm{S} 4} i_{\mathrm{S} 4}$

where $P_{\text {full }}, P_{\mathrm{S} 1}, P_{\mathrm{S} 2}, P_{\mathrm{S} 3}$, and $P_{\mathrm{S} 4}$ are the mean probabilities of the channel dwelling in each conducting state (full open and $\mathrm{S} 1-\mathrm{S} 4)$ and $i_{\text {full }}, i_{\mathrm{S} 1}, i_{\mathrm{S} 2}, i_{\mathrm{S} 3}$, and $i_{\mathrm{S} 4}$ are the mean current amplitudes for the corresponding conductance levels. Average currents were computed for recordings containing a singlechannel gating at holding potentials of +30 and $-30 \mathrm{mV}$.

Mean-variance histograms were computed using the method described by Patlak [18] and implemented in C and Python, from traces digitally filtered (Gaussian Filter) at $800 \mathrm{~Hz}$ and using at least $1 \mathrm{~min}$ of continuous recording. Briefly, a window of given length was stepped along the data, producing a pair of mean and variance values for each window location. These co-ordinate pairs were then binned in two-dimensions producing the histogram. For the histograms presented here, a window length of $10 \mathrm{~ms}$ was used.

SDS-polyacrylamide gel electrophoresis and immunoblot analysis

To examine the distribution of TRIC subtypes in the mouse muscle SR, total microsomes were prepared from WT mice and then separated by discontinuous sucrose density gradient centrifugation to yield the surface membrane (sarcolemma and transverse tubule-rich), longitudinal SR-rich (LSR) and junctional SR-rich (HSR) fractions as previously described [23]. Various fractions were subjected to SDS-polyacrylamide gel electrophoresis and Western blotting using previously described methods [20, 32] as required. Antibodies to TRICA, TRIC-B, and Junctophilin type 1 (JP1) were produced 
inhouse and the antibody for RyR was purchased from Thermo Fisher Scientific Inc. (Rockford, USA).

Statistics

Data are expressed as mean $\pm \mathrm{SD}$, where $n=3$, or mean $\pm \mathrm{SEM}$, where $n \geq 4$. Differences between mean values were assessed using a Student's $t$ test. A $p<0.05$ was taken as significant.

Materials

All chemicals were purchased from VWR (Poole, UK) or Sigma-Aldrich (Dorset, UK). All solutions were prepared in MilliQ deionised water (Millipore, Harrow, UK), and those for use in bilayer experiments were filtered through a membrane with $0.45-\mu \mathrm{m}$ pore diameter (Millipore, Harrow, UK).

\section{Results}

Figure 1 shows representative recordings of the $\mathrm{K}^{+}$current fluctuations that are observed when LSR vesicles isolated from the heart and skeletal muscle of WT mice are incorporated into artificial bilayers in symmetrical solutions of $210 \mathrm{mM}$ KPIPES. These single-channel events look similar to those obtained from cardiac or skeletal SR vesicles of other species, including rabbit, sheep, dog, and bullfrog [3, $4,7-10,15,16,20,26,28]$. Channel gating was characterised by openings to relatively long-lived full open states and by transitions to other sub-conducting open states that often look 'messy' or noisy. The full open state was $211 \pm 17 \mathrm{pS}(\mathrm{SD} ; n=6)$ for channels derived from skeletal SR and $200 \pm 5 \mathrm{pS}$ (SD; $n=4)$ for those from cardiac SR. Since we know that both TRIC-A and TRIC-B are expressed in striated SR, we cannot definitively assign any channel opening as belonging to TRIC-A or TRIC-B. We have therefore used two different approaches that guaranteed the absence of the TRIC-A isoform. We have incorporated (1) the skeletal muscle LSR membrane fraction from Tric- $a$-KO mice or (2) recombinantly expressed, detergent-purified mouse TRIC-B into bilayers in symmetrical $210 \mathrm{mM}$ KPIPES.

In rabbit skeletal muscle, TRIC-A is immunochemically detected in the nuclear and SR membranes [32]. Figure 2a shows the distribution of TRIC subtypes in the mouse muscle membrane fractions. As expected from previous observations, both TRIC subtypes were slightly enriched in the HSR, but were distributed throughout the SR fractions (Fig. 2b). At the same time, because of the similar protein compositions of the microsomal preparations from WT and Tric- $a$-KO mice (Fig. 2c), SR protein expression seems roughly normal in Tric-a-KO muscle. Indeed, Western blot analysis detected regular expression of TRIC-B, RyR and
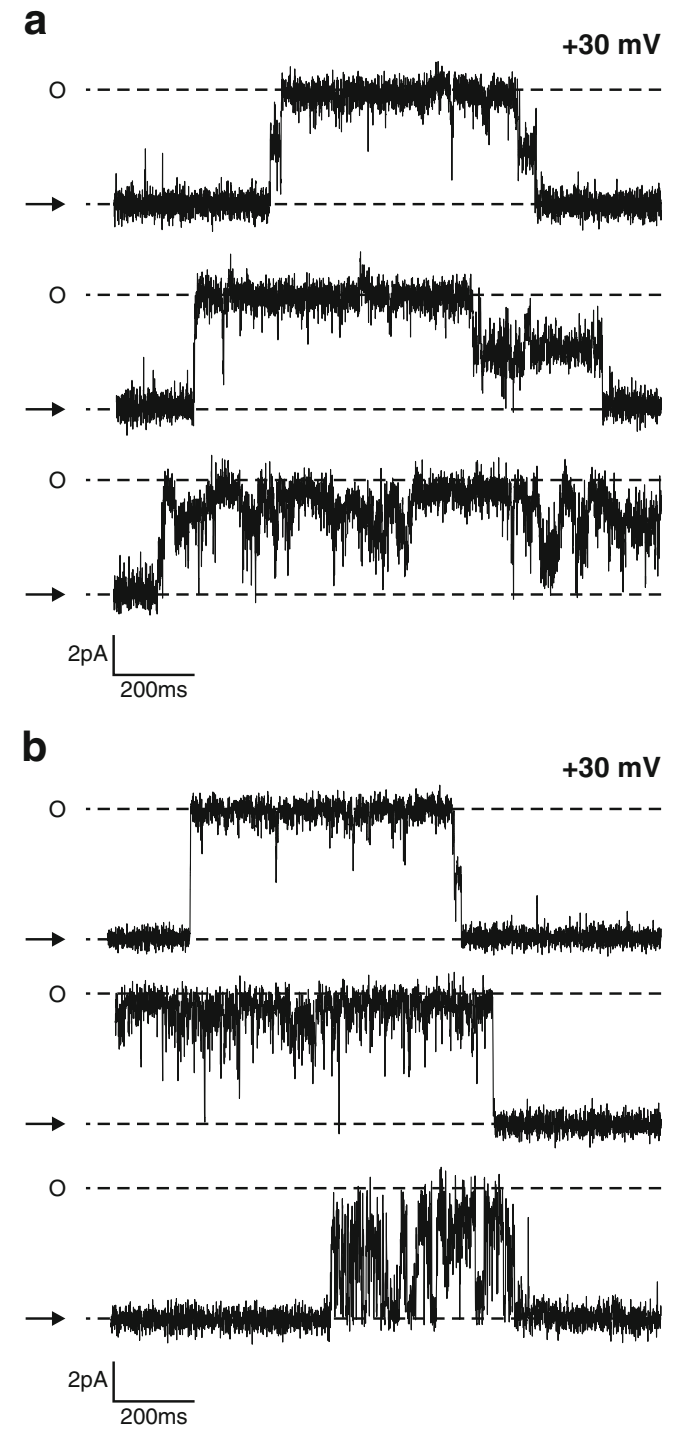

Fig. 1 Native TRIC channel currents from mouse cardiac and skeletal LSR. Examples of $\mathrm{K}^{+}$current fluctuations recorded after the incorporation of WT mouse cardiac (a) and skeletal (b) LSR membrane vesicles in symmetrical solutions of $210 \mathrm{mM}$ KPIPES. Channel openings are upward deflections, and $O$ and the black arrow indicate the open and closed channel levels, respectively

junctophilin type 1 in Tric-a-KO muscle (Fig. 2d). The SR preparations from Tric- $a$-KO mice therefore appear to provide an ideal model system from which to examine native TRIC-B channels.

When Tric-a-KO skeletal LSR vesicles were reconstituted into bilayers in order to observe native TRIC-B channel gating in isolation, we observed channel gating that was always characterised by openings to several sub-conductance states. In the event of a simultaneous incorporation of RyR channels, the record was discarded. A representative example of a native TRIC-B channel recording is illustrated in Fig. 3a. The full open channel conductance of native TRICB channels was approximately $200 \mathrm{pS}$ and was consistently 

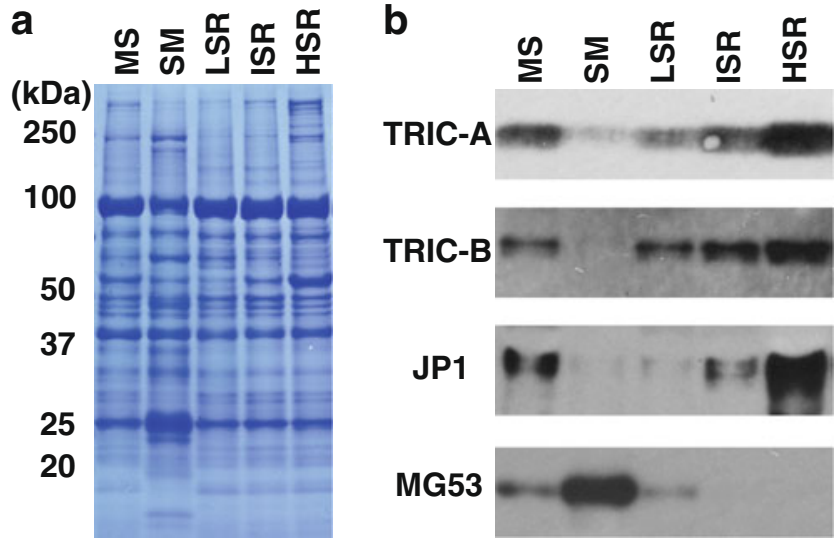

a
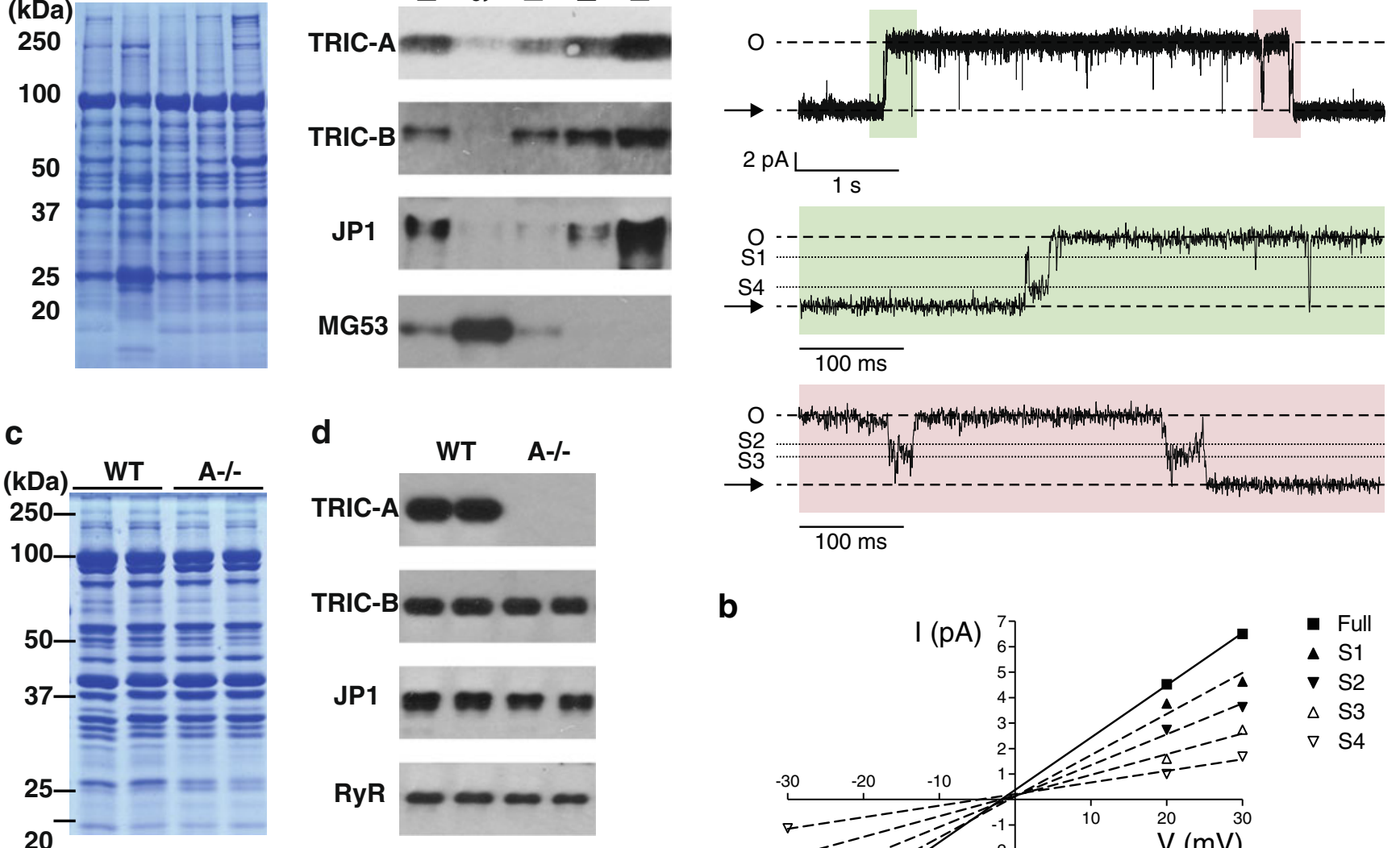

b

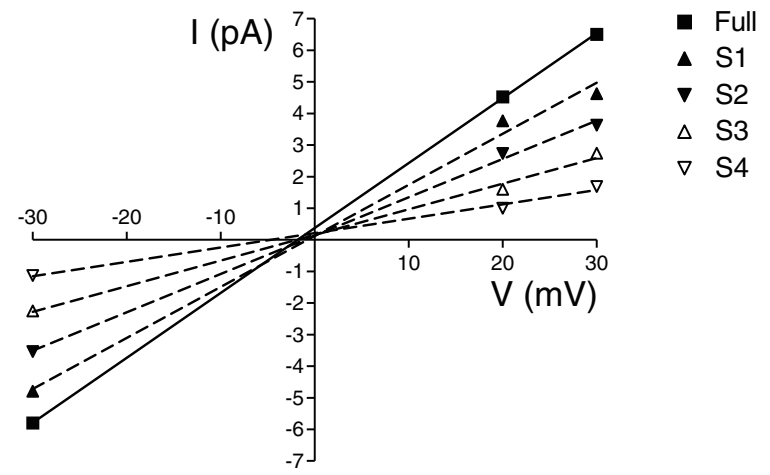

Fig. 2 SDS-PAGE analysis and immunoblot analysis of TRICsubtypes in mouse skeletal SR. a, b Distribution of TRIC-subtypes in mouse skeletal muscle membrane fractions obtained after sucrose gradient separation. The membrane fractions ( $5 \mu \mathrm{g}$ protein/lane) were separated on an SDS-polyacrylamide gel, and stained with Coomassie blue (a) or subjected to Western blot analysis (b) with antibodies to TRIC-A, TRIC-B, Junctophilin type 1 ((JP1) as a marker for HSR [25]) and MG53 (as a marker for the surface membrane fraction [1]). MS microsomes, $S M$ surface membrane fraction (sarcolemma and transverse tubule-rich), LSR light SR (longitudinal SR-rich), ISR intermediate SR, HSR heavy SR (junctional SR-rich). c, d Expression of TRIC subtypes in skeletal muscle from Tric- $a$-knockout mice. Microsomal fractions $(0.5-5 \mu \mathrm{g} /$ lane) were separated on an SDS-polyacrylamide gel and stained with Coomassie blue (c) or analysed by immunoblotting reaction (d) with antibodies to TRIC-A, TRIC-B, JP1 and RyR

observed in 34/34 experiments. This was similar to the full open state of the channels derived from WT SR (Fig. 1). Channel gating was also characterised by rapid transitions between multiple sub-conductance states. Many of these events were too brief to resolve, and so we have only measured those levels that could be detected manually. We found that the channels dwelt in four predominant subconductance levels that were approximately $80,60,46$, and $30 \%$ of the full open state. These are detailed in Table 1 . We predict that further sub-conductance states will be observed as the resolution of the single-channel events is improved. The corresponding current-voltage relationships of the full and the

Fig. 3 Native TRIC-B channel currents from mouse Tric- $a$-KO skeletal LSR. a Representative gating of a single native TRIC-B channel obtained from Tric- $a$-KO tissue. Openings are upward deflections. $O$ and the black arrow indicate the full open and the closed levels, respectively. Two sections (pink and green) of the trace are illustrated on an expanded time-scale below the original record to illustrate typical examples of sub-conductance events. The channel dwells primarily in $O$, but there are also transitions to other distinct sub-conductance states (S1-S4). b Linear regression of the current-voltage relationships for the channel shown in a yields conductances of 205 , $162,121,81$ and $45 \mathrm{pS}$ for the full open, S1, S2, S3 and S4 states, respectively

four sub-conductance levels detected are shown in Fig $3 \mathrm{~b}$ for the channel shown in Fig. 3a.

Table 1 Native TRIC-B conductance values for the full and the four main sub-conductance levels

\begin{tabular}{llllll}
\hline & Full open & S1 & S2 & S3 & S4 \\
\hline \% of full open state & 100 & 80 & 60 & 46 & 30 \\
Mean \pm SEM (pS) & $199 \pm 2$ & $161 \pm 2$ & $123 \pm 1$ & $93 \pm 2$ & $60 \pm 2$ \\
$n$ & 34 & 18 & 24 & 21 & 28 \\
\hline
\end{tabular}


When we incorporated recombinantly expressed, detergent-purified TRIC-B channels into bilayers, similar current fluctuations with multiple sub-conductance state openings to those observed in Figs 1 and 3 were observed with the exception that the channels did not appear to open to the full open state. The data are shown in Supplementary Fig. 2. We concluded that the expression system and/or purification procedure may have led to changes in protein folding and altered functional behaviour of TRIC-B channels so that they rarely open to the full open state. We therefore focused exclusively on using the native TRIC-B channels obtained from Tric- $a$-KO tissue in order to characterise the key aspects of TRIC-B gating.

To investigate whether TRIC-B is selective for cations, experiments were performed in a $\mathrm{KCl}$ gradient $\left(810 \mathrm{mM} \mathrm{K}^{+}\right.$, $600 \mathrm{mM} \mathrm{Cl}^{-}$cis; $210 \mathrm{mM} \mathrm{K}^{+}$trans, Fig 4a). A parallel shift to the left but no change in single-channel conductance was observed. The maximum conductance of the fully open channel in $\mathrm{KCl}$ gradient was $212 \pm 8 \mathrm{pS}(\mathrm{SD}, n=6)$, and the reversal potential $\left(E_{\mathrm{rev}}\right)$ was $-37.7 \mathrm{mV}$. These values are
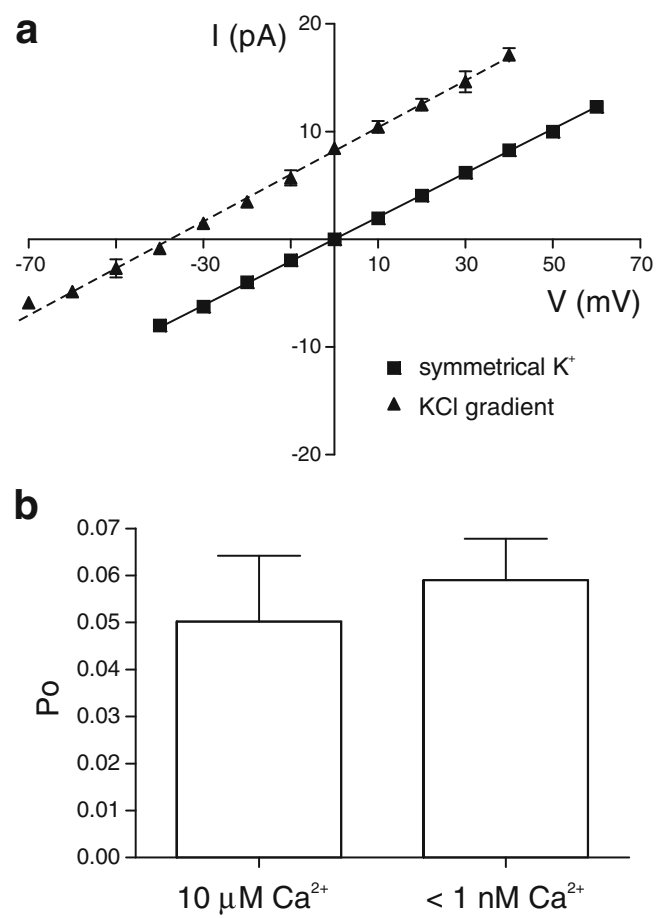

Fig. 4 Permeability properties of TRIC-B and cytosolic $\mathrm{Ca}^{2+}$ sensitivity. a Current-voltage relationships for the full open channel level of native TRIC-B under symmetrical conditions of $210 \mathrm{mM} \mathrm{K}{ }^{-}$PIPES (black line) and under gradient conditions $\left(810 \mathrm{mMK}^{+}, 600 \mathrm{mM} \mathrm{Cl}^{-}\right.$in the cis chamber and $210 \mathrm{mM} \mathrm{K}^{+}$in the trans chamber) (dashed line). Current amplitudes shown are the mean \pm SD from six experiments. Where not shown, error bars are within the symbols. Under gradient conditions, the current voltage relationship yields a reversal potential of $-37.7 \mathrm{mV}$. b Mean data illustrating the Po of the full open state of native TRIC-B channels in the presence of $10 \mu \mathrm{M}$ cytosolic free $\mathrm{Ca}^{2+}$ or with $<1 \mathrm{nM}$ free $\mathrm{Ca}^{2+}$ (after addition of $10 \mathrm{mM}$ EGTA to the cis chamber) at a holding potential of $+30 \mathrm{mV}(p>0.05$, SEM, $n=4)$ similar to the calculated value for a channel ideally selective for cations, where $E_{\text {rev }}$ is $-34 \mathrm{mV}$.

The free cytosolic $\left[\mathrm{Ca}^{2+}\right]$ was maintained at $10 \mu \mathrm{M}$ to be consistent with our previous experiments with purified and native TRIC and RyR channels. We have previously reported that purified TRIC-B channels were activated by raising cytosolic $\mathrm{Ca}^{2+}$ from 10 to $100 \mu \mathrm{M}$ [20]. To examine whether cytosolic $\mathrm{Ca}^{2+}$ is a requirement for TRIC-B activation, we lowered the free $\left[\mathrm{Ca}^{2+}\right]$ to below $1 \mathrm{nM}$ by addition of a high concentration of EGTA. No significant change in Po was observed. The mean data is shown in Fig. 4b. These experiments demonstrate that, although previous experiments show that TRIC-B channels exhibit sensitivity to changes in cytosolic $\mathrm{Ca}^{2+}$ above $10 \mu \mathrm{M}, \mathrm{Ca}^{2+}$ is not an absolute requirement for channel activation as the channels still open at free $\mathrm{Ca}^{2+}$ concentrations that are well below the resting levels found in cells.

Detailed analysis of TRIC-B gating was conducted only for recordings where a single channel was gating in the bilayer $(n=8)$. Identification of sub-conductance levels was extremely difficult in records containing multiple channels. We found that a key feature of native TRIC-B channel gating was the voltage dependence. A representative example of a TRIC-B single-channel recording at holding potentials of +30 and $-30 \mathrm{mV}$ is shown in Fig. 5a. TRIC-B Po was significantly reduced at negative holding potentials. The probability of dwelling in the full open channel level was $0.041 \pm 0.011$ at $+30 \mathrm{mV}$ but only $0.009 \pm 0.007$ at $-30 \mathrm{mV}(n=8$; SEM; $p<0.05)$.

Examples of typical sub-conductance gating of single TRIC-B channels and the corresponding amplitude histograms at +30 and $-30 \mathrm{mV}$ are illustrated in Fig. 6. For each trace, a 200-ms section of the recording is shown on an expanded time scale so that transitions to the four main sub-conductance states are easily visualised. It should be noted that TRIC-B openings at negative potentials were sporadic and that channel gating consisted primarily of transitions to sub-conductance states that did not reach the full open channel level as shown in Fig. 6e.

Mean data of the probabilities of dwelling in the full, the closed and the four sub-conductance states (S1, S2, S3 and S4) at holding potentials of +30 and $-30 \mathrm{mV}$ for the eight single channels are compared in Fig. 7a. Grouping the data in this manner suggests that the channels spend longer in the sub-conductance open states (especially S4) than in the full open state and that there was virtually no voltage dependence of gating. However, this was not what we observed for most channels. Further inspection of the data showed that two channels were heavily weighting the data and so we excluded them from the group analysis. Mean data was then obtained for the six remaining channels (channels 1-6; 
Fig. 5 Native TRIC-B

channels are voltage-dependent. a Representative single-channel recordings of native TRIC-B channels at +30 and $-30 \mathrm{mV}$.

The closed and full open channel levels are indicated by the arrow and $O$, respectively. b Mean data illustrating the Po of the full conductance open state at holding potentials of +30 and $-30 \mathrm{mV}$. TRIC-B channel activity was reduced at $-30 \mathrm{mV}$ compared to $+30 \mathrm{mV}(* p<0.05$, SEM, $n=8$ ) a
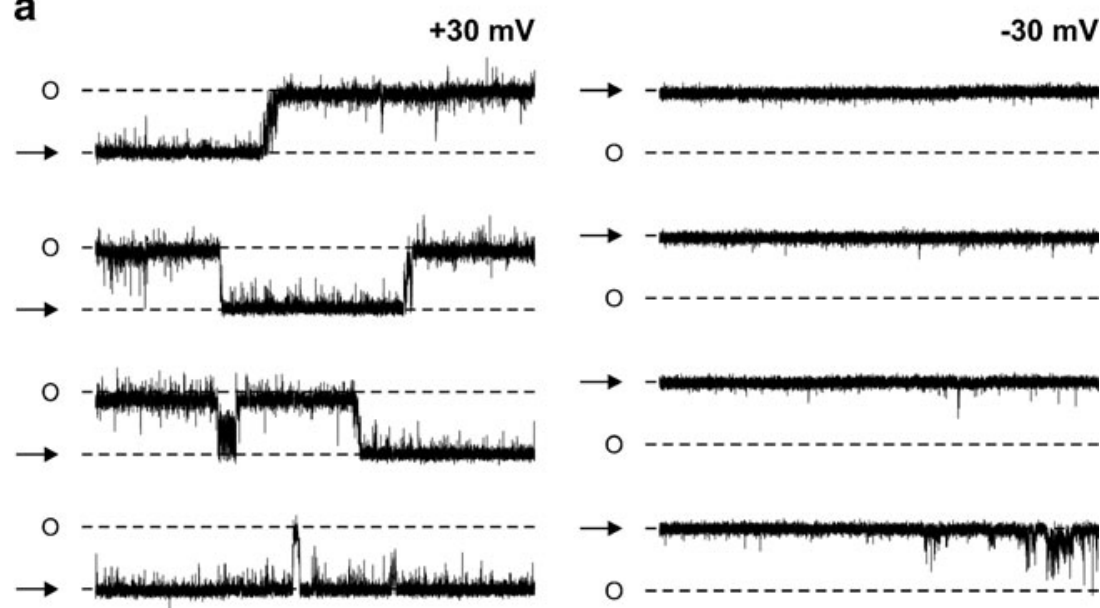

○
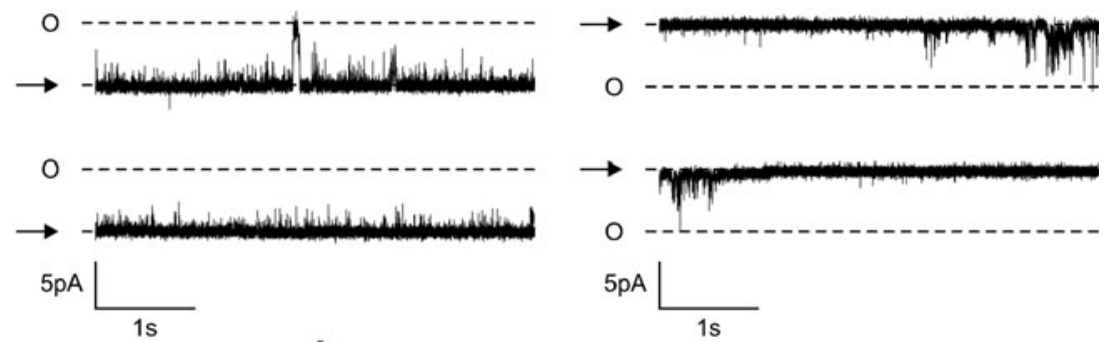

b

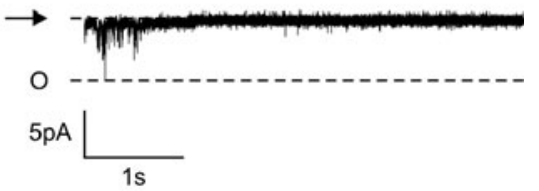

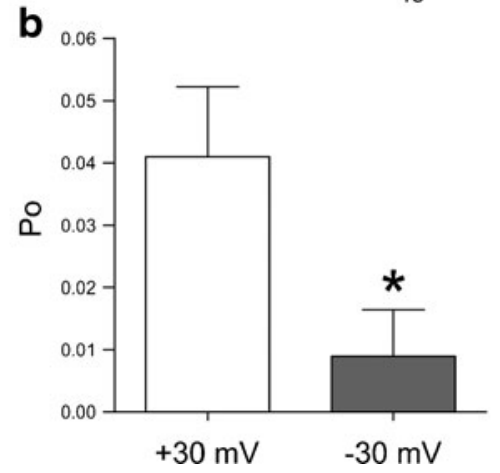

Fig. 7b), and the values for the two outliers, channels 7 and 8 , were plotted individually.

This simple analysis clearly demonstrates that the gating of TRIC-B is variable and complex and that there are at least three distinct patterns of gating. The first and most regularly observed pattern $(n=6 / 8)$ was seen in channels which, at positive potentials, exhibit a higher probability of dwelling in the full open state than in the subconductance states. These channels also exhibited strong voltage-dependent gating and were more open at +30 than $-30 \mathrm{mV}$. Importantly, the main determinant of the voltage dependence of open probability ( $\mathrm{Po}$ ) was the reduction in the time spent in the full open state. The mean data for this group of channels is shown in Fig. 7b. A second pattern of gating was observed in one of the outlying channels (channel 7). This channel was voltage dependent but dwelt for longer in the sub-conductance states (especially S4) than in the fully open state at both +30 and $-30 \mathrm{mV}$ (Fig. 7c). In this channel, the probability of dwelling in all of open states (full and sub) was reduced at $-30 \mathrm{mV}$ in comparison to $+30 \mathrm{mV}$. A third type of gating was observed in channel 8 . Like channel 7, channel 8 also dwelt for longer in the sub-conductance states (especially S4 and S3) than in the fully open state, but it was voltage independent and had very high overall Po (Fig. 7d). Sections of the single-channel record of this particular channel and the corresponding amplitude histograms illustrating the predominant gating in the lower sub-conductance states are shown in Fig. 8. It can be seen that the amplitude histogram distributions are very different to those shown in Fig. 6.

The rapid transitions between multiple sub-conductance states, together with the fact that TRIC-B channel gating does not conform to a single pattern of gating, makes it difficult to provide a simple and accurate kinetic model that encapsulates the key aspects of TRIC-B gating and that might allow us to quickly distinguish it from TRIC-A. We have therefore plotted mean-variance histograms in order to provide an accurate but also a more easily accessible and visual summary of the important aspects of TRIC-B gating. These are shown in Fig. 9a and $\mathrm{b}$ for a typical low Po channel belonging to the set of 6 channels grouped together in Fig. 7b. In Figure 9c and d, the mean-variance plots for outlier channel 8, which exhibited high Po, voltage 
a
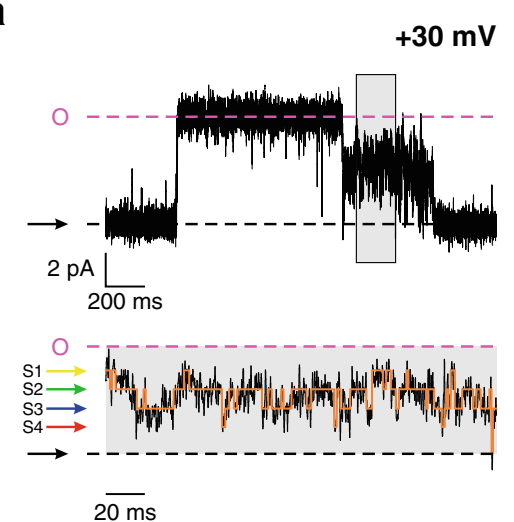

C

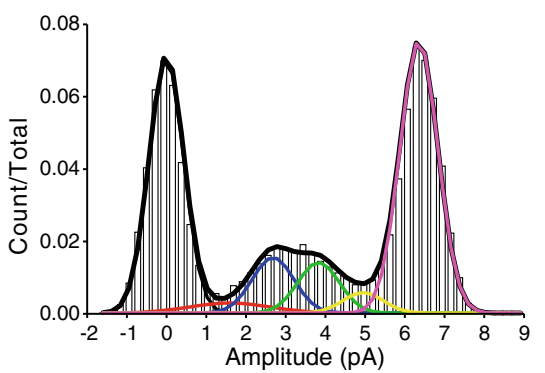

e

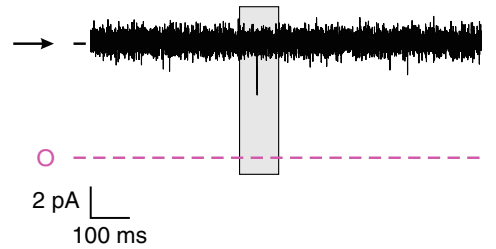

Fig. 6 Typical gating of a native TRIC-B channel at +30 and $-30 \mathrm{mV}$. a, b Long TRIC-B channel openings at $+30 \mathrm{mV}$ and at $-30 \mathrm{mV}$, respectively. The black arrow indicates the closed state; $O$ (pink) indicates the fullopen conductance of TRIC-B. Sub-conductance states $(\mathrm{Sn})$ are indicated as follows: S1 yellow, highest sub-state; S2 green; $\mathrm{S} 3$ blue; $\mathrm{S} 4$ red, lowest sub-state. Below each trace, an expanded segment $(200 \mathrm{~ms})$ of trace with SKM idealisation (orange trace) overlaid on top of the filtered data (Gaussian filter, $1 \mathrm{kHz}$ ) is shown. c, $\mathbf{d}$ All-points amplitude histograms of the recordings represented in $\mathbf{a}$ and $\mathbf{b}$, respectively. Gaussian

independence and predominance of gating in sub-states S3 and S4 are shown for comparison.

The colour density of the plots is proportional to the number of samples binned for a given mean-variance pair, and, therefore, if colour density is high (yellow) for a particular current amplitude, this reflects a high number of transitions to and from open/closed states of that current amplitude. Regions with a constant (generally low) variance and a high sample density may correspond to discrete conductances of the TRIC-B channel such as those identified and described in Table 1. Arcs through high-variance regions often correspond to transitions between states and high density in these regions indicates a large number of transitions. b
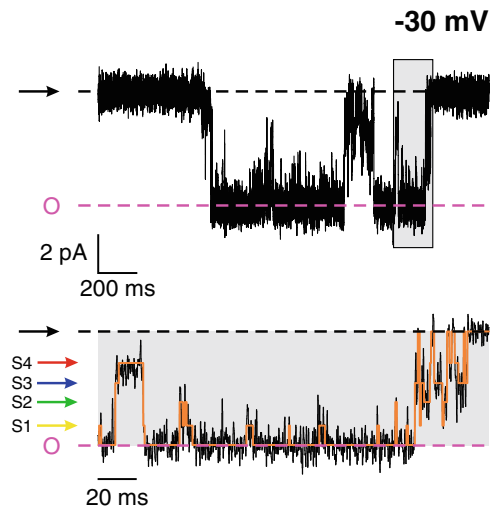

d

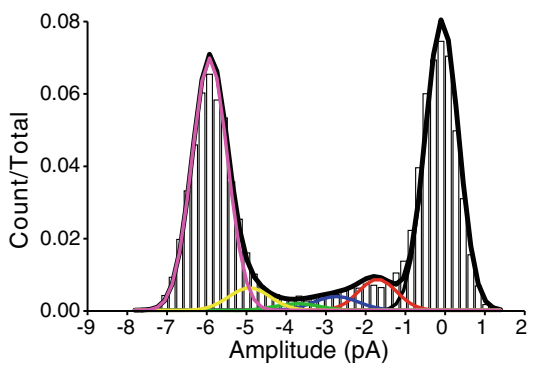

$-30 \mathrm{mV}$

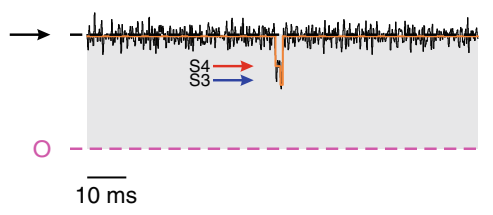

distributions computed using the SKM algorithm [21] for the closed, open and Sn states are superimposed and coloured as above. The thick black trace represents the sum of the Gaussians (the combined fit of the model's state amplitudes to the filtered data). e A typical brief TRIC-B opening detected at a holding potential of $-30 \mathrm{mV}$. The black arrow indicates the closed channel level and $O$ (pink) indicates the full open state of TRIC-B. On the bottom, an expanded section of the trace is illustrated (100 ms). In this case, the channel appears to gate only to the subconductance states S3 (blue) and S4 (red)

For the low Po, voltage-dependent channel at $+30 \mathrm{mV}$ (Fig. 9a), the points are clustered closely around the closed state, with the region of next highest density (and low variance) being the full open state. Arcs through regions of higher variance correspond to channel transitions to and from the various sub-conductance states and reflect the comparatively brief dwell times in those states. At negative potentials (Fig. 9b), the majority of time is spent in the closed state, and it is clear that there are no detected transitions to the fully open state. For channel 8 (the voltageindependent channel), the markedly different gating behaviour is clearly reflected in the corresponding mean-variance plots shown in Fig. 9c and d. At positive potentials (Fig. 9c), the channel spends proportionately more time in the 
a

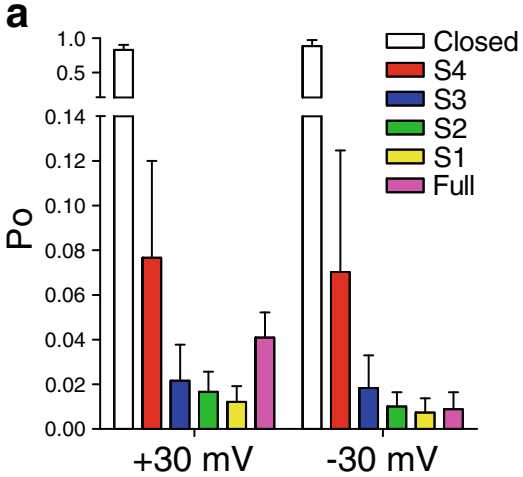

C

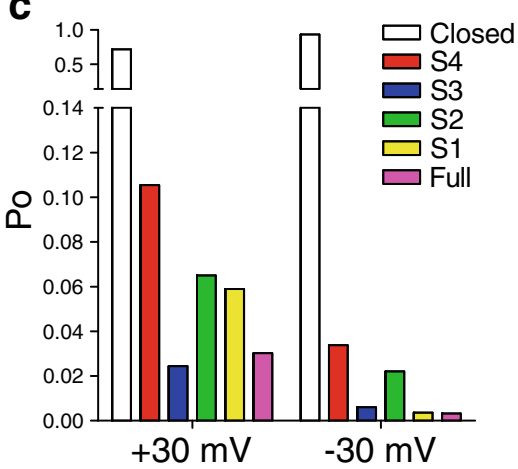

Fig. 7 Mean data of the probabilities of dwelling in the full, the closed and the four sub-conductance states. Open probabilities for individual conductance states of native TRIC-B channels at +30 and $-30 \mathrm{mV}$ are compared. The full open conductance (pink) and sub-conductance states in order of increasing current amplitude: S4 red, lowest subconductance; S3 blue; S2 green; S1 yellow, highest sub-conductance. a Mean probabilities of dwelling in each state for all 8 channels. b Mean probabilities of dwelling in each state for a sub-set of six channels taken from the set of channels in $\mathbf{a}$, which exhibit a

sub-conductance states than the channel in panels a and $b$. Transitions to and from the small conductance sub-states (S4 and S3) give rise to areas of particularly high variance for a given mean current. The large areas of high variance between the black and red and blue arrows indicate high numbers of short-lived events and rapid transitions between the closed states and S3 and S4. Likewise, at $-30 \mathrm{mV}$ (Fig. 9d), the channel is still opening to a high Po level and a considerably large proportion of the time that the channel is open is spent switching between the full open and sub-conductance states rather than dwelling at any one particular state for long periods of time.

\section{Discussion}

By knocking out expression of TRIC-A, we have been able to study the single-channel behaviour of native mouse skeletal TRIC-B channels. This has allowed a detailed study of the biophysical characteristics of this channel without
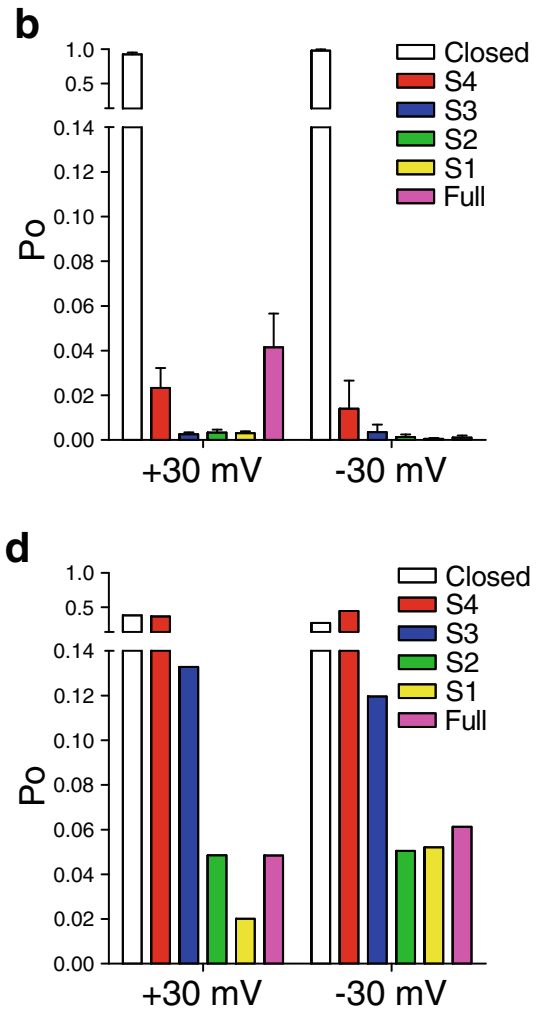

similar voltage-dependence of gating and a high probability of dwelling in the full open state (channels 1-6). c Probabilities of dwelling in each state for a single channel (channel 7) taken from the set of channels in a. This channel exhibits a high proportion of subconductance gating (Po for S4, S2, S1 is higher than for O). The channel still exhibits a degree of voltage dependence in its gating, visible for all conductance states. d Probabilities of dwelling in each state for a single channel (channel 8) taken from the set of channels in a. This channel exhibits high Po and voltage-independent gating

misclassifying any single-channel current fluctuations as TRIC-A events. Unexpectedly, we find that the full open state conductance of native mouse TRIC-B (199 pS) is significantly higher than our measurements of the highest conductance open events of purified recombinant TRIC-B (which varies between 140-160 pS under identical recording conditions (see Supplementary Fig. 2)). Instead, the full open state conductance of native TRIC-B is similar to our reported values for purified recombinant TRIC-A (192 pS) [20]. It is also similar to the highest conductance open state that we observe when we incorporate WT mouse SR into bilayers $(200 \mathrm{pS})$.

We attribute the apparent lower conductance of purified TRIC-B to modification of the gating behaviour of the channel by the purification procedure and/or the use of a non-mammalian expression system. It appears that the purified TRIC-B channels gate only in sub-conducting open states. We used CHAPS as the detergent in this study but have previously developed protocols using two other detergents ( $n$-dodecyl beta-D-maltopyranoside [20] and 
a
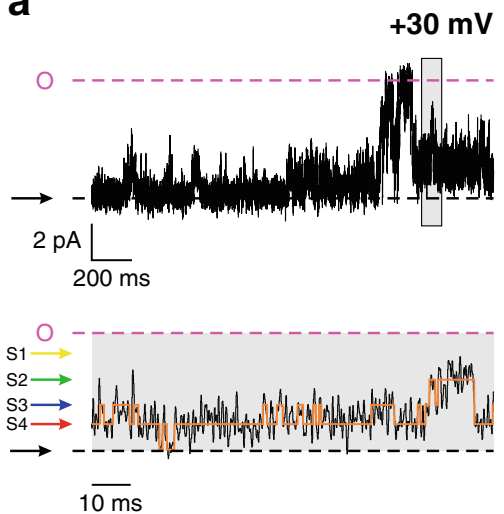

C

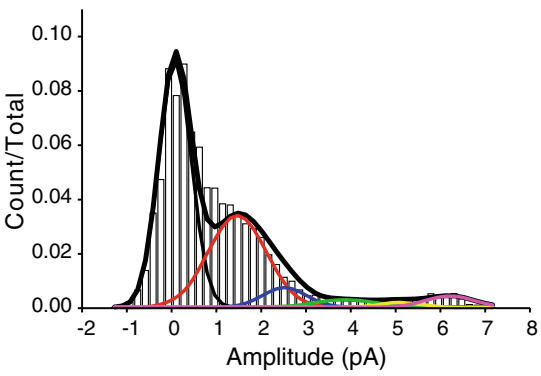

Fig. 8 Typical current fluctuations through the voltage-independent channel. a, b Representative sections of the voltage-independent, high Po TRIC-B channel (channel 8 ) recording at +30 and $-30 \mathrm{mV}$. The black arrow indicates the closed channel level (dashed black line) while the full open conductance is indicated by $O$ (pink). Sub-conductance states, in order of increasing current amplitude, are represented by S4 (red), S3 (blue), S2 (green) and S1 (yellow). Expanded sections (200 ms) show

n-decyl-beta-D-maltopyranoside (unpublished observations)), but in all cases, recovery of functional ion channels was problematic and we observed only low conductance events $(\leq 160 \mathrm{pS})$. Aside from the problems associated with protein purification, E. coli and yeast cells may not be appropriate expression systems for TRIC channels and may lead to incorrect protein folding, which does not support normal channel gating behaviour. These experiments highlight the need to study native channels.

Our experiments also revealed that TRIC-B gates to a number of sub-conductance gating states. With the resolution of single-channel events possible at the holding potentials used $(+30$ and $-30 \mathrm{mV})$, we identified four frequently visited sub-conductance gating states. With improved resolution, it is likely that additional sub-conductance levels will be revealed. We were not able to hold at higher potentials for long periods of time in order to improve the signal/noise ratio as this frequently broke the bilayers. It was usual for multiple channels to incorporate into the bilayers, but in eight experiments, we found that only single channels were active in the bilayer and we used these experiments to analyse the gating behaviour of TRIC-B channels in more detail. Previous reports suggest that $\mathrm{SR} \mathrm{K}^{+}$channels exhibit b
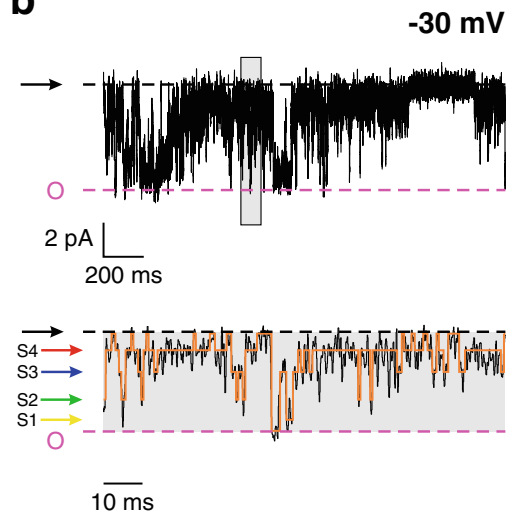

d

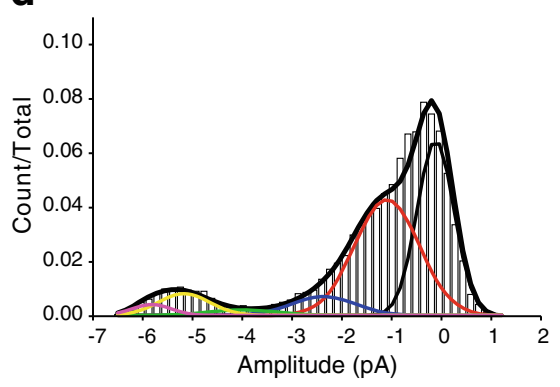

the sub-conductance gating behaviour with SKM idealization overlaid (orange). c, d All-points amplitude histograms of the portions of trace shown in a and $\mathbf{b}$, respectively. Gaussian distributions computed in SKM fit for the closed, the open and Sn states, are superimposed and coloured as above. The thick black trace represents the sum of Gaussians and the combined fit of the model's state amplitudes to the filtered data

a single sub-conductance state of approximately $60-70 \%$ of the fully open state. This sub-state has often been described as messy or noisy and there are quite wide variations in the reported amplitudes of the sub-conductance state $[6,10,15$, $19,22,26,27]$. We suggest that the noisy gating of this reported 60-70\% sub-conductance state actually reflects the rapid transitions between multiple sub-conductance states of the TRIC channels (see Figs. 6 and 8 for examples). When normal or WT SR is incorporated into bilayers, we assume that both TRIC subtypes could be incorporated. It is feasible that most previous recordings were of TRIC-A channels, since estimated TRIC-A levels are roughly 10 times more than TRIC-B levels in cardiac and skeletal SR preparations based on quantitative reverse transcriptase polymerase chain reaction (RT-PCR) and Western blotting data (D. Y. and H. T., unpublished observations). However, future work investigating the biophysical properties of native TRIC-A channels is required to address this issue.

Our experiments suggest that we may not be able to distinguish between TRIC-A and TRIC-B on the basis of single-channel conductance measurements, and this led us to attempt to define the hallmark features of TRIC-B gating. However, although there are major trends in gating 
a

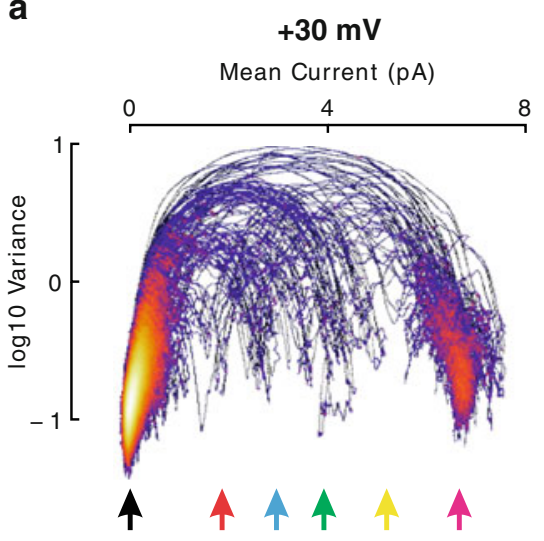

C

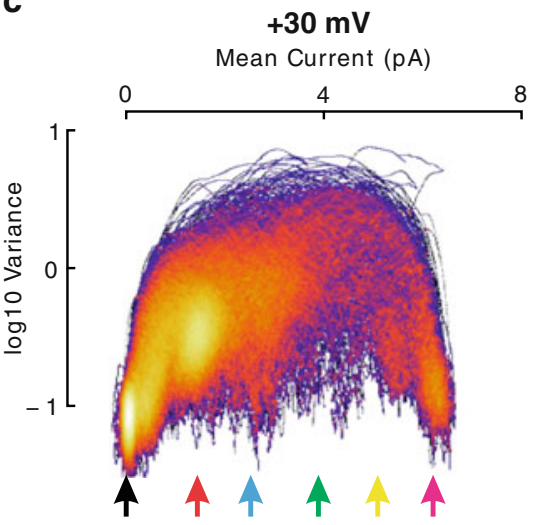

b

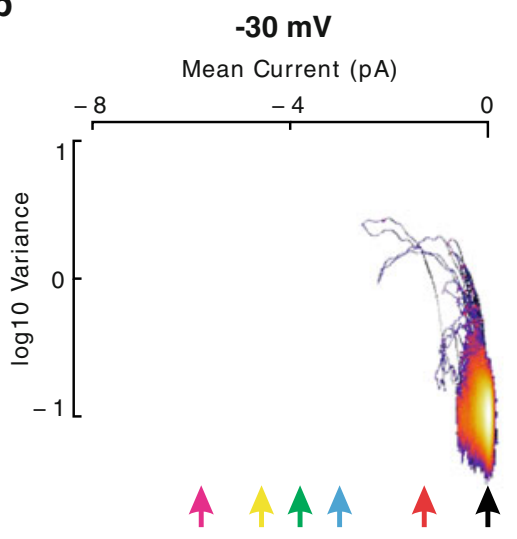

d

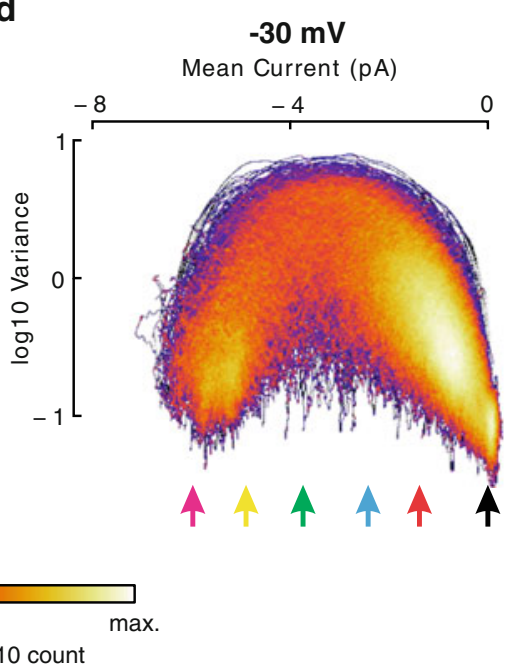

Fig. 9 Representative mean-variance histograms showing the subconductance gating behaviour exhibited by TRIC-B channels. The colour density is proportional to the number of samples binned for a given mean-variance pair. The regions with a constant (generally low) variance and a high sample density may correspond to discrete conductances of the TRIC-B channel. Arcs through high-variance regions often correspond to transitions between states and high density in these regions indicates a large number of transitions. The arrows indicate the closed (black), open (pink) and sub-conductance S1-S4 (yellow, green, blue and red, respectively) state levels that were measured in the corresponding single-channel traces. a, b Typical histograms for a channel belonging to the set of channels grouped together in Fig. 7b (channels 1-6). These channels were characterised by voltagedependent gating and higher Po in the full open state than in the subconductance states. At $+30 \mathrm{mV}$, the channel is closed $(0 \mathrm{pA})$ most of the time with some brief transitions to various sub-states. These transitions manifest themselves as arcs through high-variance regions;

behaviour it is evident that TRIC-B gating is extremely variable and that a single unified model of gating would not be adequate. In terms of hallmark features, voltage dependence was the most obvious trait, and yet this was not observed for all channels. The majority of channels (7/8) channels were strongly voltage dependent with very low Po values at $-30 \mathrm{mV}$, but one channel was clearly not regulated by voltage and exhibited high Po at both $\pm 30 \mathrm{mV}$. Another however, because they are relatively infrequent, the individual subconductance states are not defined by clear high-density regions. For this channel, it can be seen that the majority of the openings are to the full open state. Conversely, at $-30 \mathrm{mV}$, the samples are almost exclusively distributed around the closed state, with a small bias towards S4 (red arrow), the lowest conductance open state. There are no openings to the full open state. $\mathbf{c}, \mathbf{d}$ Voltage-independent gating of channel 8 . The channel dwells for a noticeably longer proportion of time in subconductance open states than the full open state at both +30 and $-30 \mathrm{mV}$. The samples in the mean-variance plot are clustered with similar density around regions with low variance representing mean currents that correspond to sub-conductance states. Rapid gating between closed, S4 and S3 states gives rise to a slightly higher variance region for a given mean than would be expected for long sojourns in a specific conductance state. The density of samples around the open states is much higher than in $\mathbf{a}$ and $\mathbf{b}$, corresponding to a much higher overall Po

prevalent feature was the dominance of the full open state at $+30 \mathrm{mV}$; the Po of six in eight channels was primarily determined by openings to the fully open state, and only two of the eight channels opened predominantly to the subconductance states. It is possible that the channels that appear to be outliers (shown in Fig 7c and d) may simply represent examples of the wide range of gating behaviour that occurs physiologically. 


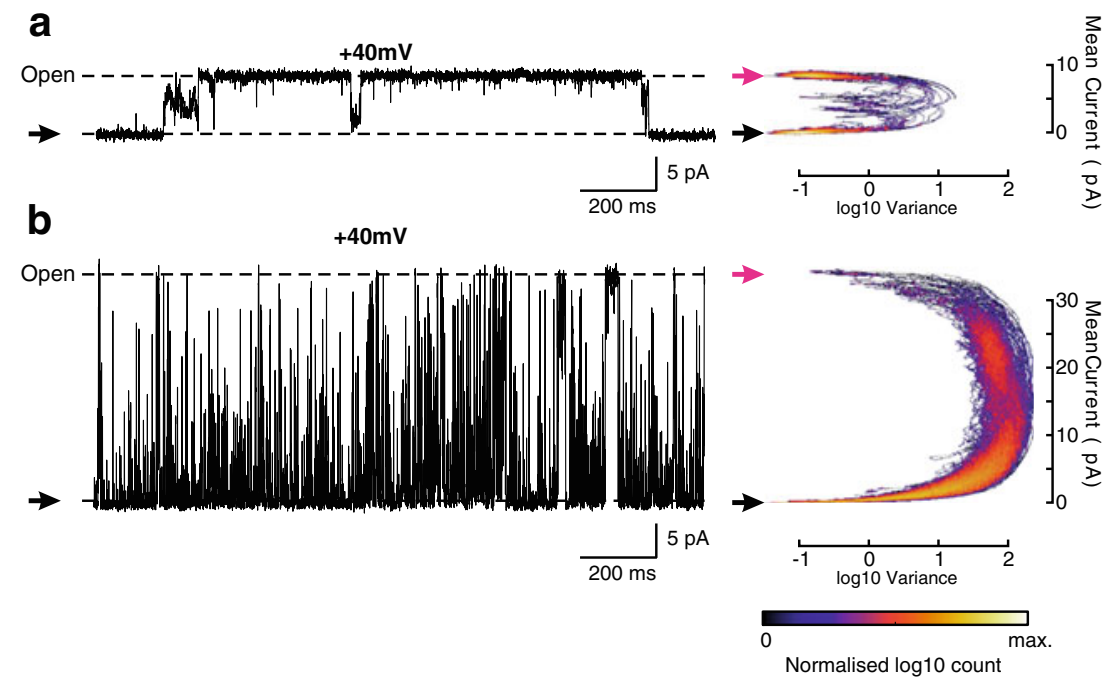

Fig. 10 Comparison of the mean-variance histograms derived from single-channel recordings of TRIC-B and RyR from mouse skeletal LSR. Representative current fluctuations for each channel are shown to the left of the mean-variance histograms, illustrating: a the typical gating of a voltage-dependent TRIC-B channel. The channel opens predominantly to the full open state, with brief, but obvious, sojourns to sub-conductance states. b The gating of a typical, single RyR channel. Gating consists primarily of rapid gating between the closed and fully open state of the channel but there are also some infrequent, longer dwell-times in the fully open state. Histogram colour intensity is proportional to the sample density. The mean-variance histograms are rotated $90^{\circ}$ in comparison to those in Fig. 9. This is to highlight the relationship of the mean-variance histograms to the single-channel openings. The black and pink arrows indicate the closed and fully open channel current amplitudes, respectively, and the corresponding

We have used mean-variance plots as a method of highlighting the important aspects of TRIC-B gating in a snapshot. This approach allows us to visualise not only the approximate Po of the channel but also the preponderance of sub-conductance state gating and the frequency of transitions between certain states. The plots demonstrate how the usual gating of TRIC-B that is observed in $75 \%$ of the channels (six of eight) is dominated by openings to the fully open state at $+30 \mathrm{mV}$ but by sub-state gating at $-30 \mathrm{mV}$ (Fig. 9a, b). Similarly, it is easy to observe the higher Po and frequent transitions between sub-conductance states at both +30 and $-30 \mathrm{mV}$ for the channel data shown in Fig. 9c and $\mathrm{d}$. Our goal is to use this new method of visualising

Table 2 Average currents for the three groups of channels and the percent reduction of the average currents observed at $-30 \mathrm{mV}$

\begin{tabular}{llll}
\hline Group & \multicolumn{2}{l}{ Average current $(\mathrm{pA})$} & \\
\cline { 2 - 3 } & $+30 \mathrm{mV}$ & $-30 \mathrm{mV}$ & \% reduction at $-30 \mathrm{mV}$ \\
\hline Channels 1-6 & 0.320 & 0.051 & 84 \\
Channel 7 & 0.963 & 0.196 & 80 \\
Channel 8 & 1.598 & 1.939 & - \\
\hline
\end{tabular}

variance at these current amplitudes. The current amplitude of the full open state of RyR is much higher than that of TRIC-B, and this leads to higher overall variance in regions that correspond to rapid transitions between states. RyR gating under the experimental conditions of the recording (symmetrical $210 \mathrm{mM} \mathrm{K}, 10 \mu \mathrm{M}$ free $\mathrm{Ca}^{2+}$ ) is typified by rapid transitions between the closed and fully open channel levels that are too brief to resolve and which are truncated by filtering. This gives rise to histograms with arcs of high density and high variance. Qualitatively, this type of gating behaviour is clearly different to the sub-conductance gating activity of TRIC-B, where longer-lived subconductance states can be distinguished from transitions between states by their lower overall variance and discrete regions of higher density. For both TRIC-B and RyR, the holding potential was $+40 \mathrm{mV}$. The data was sampled at $100 \mathrm{kHz}$ and filtered at $800 \mathrm{~Hz}$ using $65-80 \mathrm{~s}$ of continuous recording

TRIC channel gating to compare and contrast the gating behaviour of TRIC-A and TRIC-B channels. We will also be able to assess how regulators of TRIC channels modify gating without building complicated kinetic gating models that are subject to error because of the poor resolution of the brief sub-conductance events.

As yet, we do not have opportunity to work with native TRIC-A channels since the Tric- $b$-KO mouse is not viable. Figure 10, however, illustrates how mean-variance plots can be an effective method of highlighting differences in singlechannel conductance, gating behaviour and the prevalence

Table 3 Contribution of sub-conductance state gating to the average current for the three groups of channels

\begin{tabular}{lllll}
\hline Group & \multicolumn{3}{l}{$\begin{array}{l}\text { Average current attributed only to sub-state } \\
\text { gating (pA) }\end{array}$} \\
\cline { 2 - 5 } & $+30 \mathrm{mV}$ & $\begin{array}{l}\text { \% of total } \\
\text { current }\end{array}$ & $-30 \mathrm{mV}$ & $\begin{array}{l}\text { \% of total } \\
\text { current }\end{array}$ \\
\hline Channels 1-6 & 0.075 & 23 & 0.045 & 88 \\
Channel 7 & 0.782 & 81 & 0.177 & 90 \\
Channel 8 & 1.310 & 82 & 1.572 & 81 \\
\hline
\end{tabular}


of sub-conductance gating states. The figure compares mean-variance plots from single-channel recordings of TRIC-B (top) and RyR (bottom). Note, for example, the high conductance and the very brief openings of RyR channels that are truncated by filtering in comparison to the obvious sub-conductance gating of TRIC-B.

In a cellular context, how would sub-conductance gating contribute to the overall currents that would flow across the SR membrane through TRIC-B? Since we have no reason to suggest that channels 7 and 8 (although atypical for our group of 8 channels) do not form part of the physiological spectrum of TRIC-B channels, how important is their contribution? To answer these questions it is useful to think about average channel current rather than Po in states.

The average TRIC-B current for a channel can be calculated from the current amplitude of the full and subconductance states and the Po in those states. Table 2 compares the average current measurements for the different groups of channels (channels 1-6, channel 6 and channel 7). These data show that channels 1-6 have low current overall in comparison to channels 7 and 8 , and exhibit an approximately $80 \%$ reduction in current at negative voltages. Channels 7 and 8 would provide most of the current across the membrane at positive potentials if this group of channels was representative of the TRIC-B channel population in the SR or ER of a cell. Channel 8 would also provide significant current at negative holding potentials and markedly alter the overall voltage dependence of the eight channels as a group.

Table 3 shows the contribution of the sub-conductance states to the average channel current. Sub-conductance state gating was low for channels $1-6$, contributing only $23 \%$ of the overall current at $+30 \mathrm{mV}$, whereas sub-state gating accounted for approximately $80 \%$ of the average current for channels 7 and 8 . For all channels, sub-conductance state gating contributed to approximately $80 \%$ of the total gating at $-30 \mathrm{mV}$. Thinking about the gating of the channels in this manner shows that the outlying channels would contribute significantly to monovalent flux across the membrane and to the overall voltage-dependence of the TRIC-B population of channels. On average, TRIC-B channels would be activated by the development of negative charge in the SR lumen with respect to the cytosol and therefore would be ideally suited to pass counter current to help balance the negative charge that would otherwise develop in the SR during a $\mathrm{Ca}^{2+}$ release event. On the other hand, a few voltage-independent channels of high Po such as channel 8 would ensure that there is always a conduit for equilibration of charge across the SR irrespective of voltage. We know very little of how TRIC channels are regulated. It is possible that the variability in TRIC-B gating behaviour that we observe could be due to heterogeneity of channels in regard to post-translational events such as phosphorylation or nitrosylation or maybe due to the presence or absence of a closely associated protein. As such, these may be physiological/pathophysiological mechanisms for stimulating or inhibiting TRIC-B channel opening or inducing sub-conductance state gating.

In summary, we show that native TRIC-B channels are voltage-dependent $\mathrm{K}^{+}$channels that exhibit complex and variable gating behaviour. The biophysical properties of TRIC-B indicate that these channels would be well suited to provide monovalent counter current in response to loss of positive charge from the SR during the $\mathrm{Ca}^{2+}$ release process. Further studies are required to characterise the singlechannel properties of native TRIC-A to identify any key differences between the TRIC channel subtypes that may be important physiologically.

Acknowledgements This study was supported by the British Heart Foundation, the Engineering and Physical Sciences Research Council and the Japan Society for the Promotion of Science

Open Access This article is distributed under the terms of the Creative Commons Attribution License which permits any use, distribution, and reproduction in any medium, provided the original author(s) and the source are credited.

\section{References}

1. Cai C, Masumiya H, Weisleder N, Matsuda N, Nishi M, Hwang M, Ko JK, Lin P, Thornton A, Zhao X, Pan Z, Komazaki S, Brotto M, Takeshima H, Ma J (2009) MG53 nucleates assembly of cell membrane repair machinery. Nat Cell Biol 11(1):56-64. doi: $10.1038 /$ ncb 1812

2. Chung SH, Moore JB, Xia L, Premkumar LS, Gage PW (1990) Characterization of single channel currents using digital signal processing techniques based on hidden Markov models. Phil Trans Roy Soc London B Biol Sci 329(1254):265-285. doi:10.1098/rstb.1990.0170

3. Coronado R, Latorre R (1982) Detection of $\mathrm{K}^{+}$and $\mathrm{Cl}^{-}$channels from calf cardiac sarcolemma in planar lipid bilayer membranes. Nature 298(5877):849-852

4. Coronado R, Rosenberg RL, Miller C (1980) Ionic selectivity, saturation, and block in a $\mathrm{K}^{+}$-selective channel from sarcoplasmic reticulum. J Gen Physiol 76:425-446

5. Forney GD Jr (1973) The viterbi algorithm. Proc IEEE 61(3):268278. doi:10.1109/proc. 1973.9030

6. Fox JA (1985) Conductance and selectivity properties of a substate of the rabbit sarcoplasmic reticulum channel. Biophys J 47:573-576. doi:10.1016/S0006-3495(85)83953-9

7. Garcia AM, Miller C (1984) Channel mediated monovalent cationfluxes in isolated sarcoplasmic reticulum vesicles. J Gen Physiol 83:819-839

8. Hill JA Jr, Coronado R, Strauss HC (1989) Potassium channel of cardiac sarcoplasmic reticulum is a multi- ion channel. Biophys $\mathrm{J}$ 55:35-45

9. Labarca P, Coronado R, Miller C (1980) Thermodynamic and kinetic studies of the gating behaviour of a $\mathrm{K}^{+}$-selective channel from the sarcoplasmic reticulum membrane. J Gen Physiol 76:397-424

10. Labarca P, Miller C (1981) A K $\mathrm{K}^{+}$-selective, three-state channel from fragmented sarcoplasmic reticulum of frog leg muscle. J Membr Biol 61:31-38 
11. Lai FA, Erickson HP, Rousseau E, Liu QY, Meissner G (1988) Purification and reconstitution of the $\mathrm{Ca}$ release channel from skeletal muscle. Nature 331:315-319

12. Latorre R, Vergara C, Hidalgo C (1982) Reconstitution in planar lipid bilayers of a $\mathrm{Ca}^{2+}$-dependent $\mathrm{K}^{+}$channel from transverse tubule membranes isolated from rabbit skeletal muscle. Proc Natl Acad Sci 79(3):805-809

13. Lindsay ARG, Williams AJ (1991) Functional characterisation of the ryanodine receptor purified from sheep cardiac muscle sarcoplasmic reticulum. Biochim Biophys Acta 1064:89-102

14. Liu QY, Lai FA, Rousseau E, Jones RV, Meissner G (1989) Multiple conductance states of the purified calcium release channel complex from skeletal sarcoplasmic reticulum. Biophys J 55:415-424

15. Liu QY, Lai FA, Shen WK, Meissner G, Strauss HC (1991) Reconstitution of the solubilized cardiac sarcoplasmic reticulum potassium channel: identification of a putative M r $80 \mathrm{kDa}$ polypeptide constituent. FEBS Lett 291:13-16

16. Miller C (1978) Voltage-gated cation conductance channel from fragmented sarcoplasmic reticulum: steady-state electrical properties. J Membr Biol 40:1-23

17. Moczydlowski E, Latorre R (1983) Gating kinetics of Ca-activated $\mathrm{K}$-channels from rat muscle incorporated into planar lipid bilayers. Evidence for 2 voltage-dependent $\mathrm{Ca}^{2+}$ binding reactions. J Gen Physiol 82:511-542

18. Patlak JB (1993) Measuring kinetics of complex single ion channel data using mean-variance histograms. Biophys J 65:29-42

19. Picard L, Côté K, Teijeira J, Greentree D, Rousseau E (2002) Sarcoplasmic reticulum $\mathrm{K}(+)$ channels from human and sheep atrial cells display a specific electro-pharmacological profile. J Mol Cell Cardiol 34(9):1163-1172

20. Pitt SJ, Park KH, Nishi M, Urashima T, Aoki S, Yamazaki D, Ma J, Takeshima H, Sitsapesan R (2010) Charade of the SR K ${ }^{+}$-channel: two ion-channels, TRIC-A and TRIC-B, masquerade as a single $\mathrm{K}^{+}$channel. Biophys J 99(2):417-426. doi:10.1016/j.bpj.2010.04.051

21. Qin F (2004) Restoration of single-channel currents using the segmental k-means method based on hidden Markov modeling. Biophys J 86(3):1488-1501. doi:10.1016/S0006-3495(04)74217-4

22. Rousseau E, Chabot H, Beaudry C, Muller B (1992) Reconstitution and regulation of cation-selective channels from cardiac sarcoplasmic reticulum. Mol Cell Biochem 114:109-117

23. Saito A, Seiler S, Chu A, Fleischer S (1984) Preparation and morphology of sarcoplasmic reticulum terminal cisternae from rabbit skeletal muscle. J Cell Biol 99:875-885
24. Sitsapesan R, Montgomery RAP, MacLeod KT, Williams AJ (1991) Sheep cardiac sarcoplasmic reticulum calcium release channels: modification of conductance and gating by temperature. J Physiol 434:469-488

25. Takeshima H, Komazaki S, Nishi M, Iino M, Kangawa K (2000) Junctophilins: a novel family of junctional membrane complex proteins. Mol Cell 6(1):11-22

26. Tomlins B, Williams AJ (1986) Solubilisation and reconstitution of the rabbit skeletal muscle sarcoplasmic reticulum $\mathrm{K}^{+}$channel into liposomes suitable for patch clamp studies. Pflugers Arch 407:341-347

27. Tomlins B, Williams AJ, Montgomery RAP (1984) The characterization of a monovalent cation selective channel of mammalian cardiac muscle sarcoplasmic reticulum. J Membr Biol 80:191-199

28. Uehara A, Yasukochi M, Imanaga I (1994) Calcium modulation of single SR potassium channel currents in heart muscle. J Mol Cell Cardiol 26:195-202. doi:10.1006/jmcc.1994.1022

29. Williams AJ (1992) Ion conduction and discrimination in the sarcoplasmic reticulum ryanodine receptor/calcium-release channel. J Muscle Res Cell Motil 13:7-26

30. Yamazaki D, Komazaki S, Nakanishi H, Mishima A, Nishi M, Yazawa M, Yamazaki T, Taguchi R, Takeshima H (2009) Essential role of the TRIC-B channel in $\mathrm{Ca}^{2+}$ handling of alveolar epithelial cells and in perinatal lung maturation. Development 136:23552361. doi:10.1242/dev.036798

31. Yamazaki D, Tabara Y, Kita S, Hanada H, Komazaki S, Naitou D, Mishima A, Nishi M, Yamamura H, Yamamoto S, Kakizawa S, Miyachi H, Miyata T, Kawano Y, Kamide K, Ogihara T, Hata A, Umemura S, Soma M, Takahashi N, Imaizumi Y, Miki T, Iwamoto T, Takeshima H (2011) TRIC-A channels in vascular smooth muscle contribute to blood pressure maintenance. Cell Metab 14 (2):231-241. doi:10.1016/j.cmet.2011.05.011

32. Yazawa M, Ferrante C, Feng J, Mio K, Ogura T, Zhang M, Lin PH, Pan Z, Komazaki S, Kato K, Nishi M, Zhao X, Weisleder N, Sato C, Ma J, Takeshima H (2007) TRIC channels are essential for $\mathrm{Ca}^{2+}$ handling in intracellular stores. Nature 448:78-82. doi:10.1038/ nature 05928

33. Zhao X, Yamazaki D, Park KH, Komazaki S, Tjondrokoesoemo A, Nishi M, Lin P, Hirata Y, Brotto M, Takeshima H, Ma J (2010) $\mathrm{Ca}^{2+}$ overload and sarcoplasmic reticulum instability in tric-a null skeletal muscle. J Biol Chem 285(48):37370-37376. doi:10.1074/ jbc.M110.170084 